\title{
Effects of control on choice of reward or punishment
}

\author{
WILLIAM J. THOMSON \\ University of Michigan, Dearborn, Michigan
}

\begin{abstract}
Previous research has indicated that, in choice situations, subjects prefer control to lack of control, and also that reward is preferred over punishment. The present study utilized 64 adult human subjects who were given two concept identification tasks to solve. The problems were either solvable (S) or unsolvable $(\overline{\mathbf{S}})$, and subjects were given feedback in the form of pokerchip reward (C) for correct responses or noise punishment (N) for errors. Four groups were given varied combinations of problem solvability and feedback on the initial two problems: (1) $S$ problem $+C$ feedback and $\mathbf{S}$ problem $+\mathrm{N}$ feedback, (2) S problem $+\mathrm{N}$ feedback and $\overline{\mathrm{S}}$ problem $+\mathrm{C}$ feedback, (3) S problem + C feedback and S problem $+N$ feedback, (4) S problem $+C$ feedback and $\bar{S}$ problem $+\mathbf{N}$ feedback. All groups were then given a third unsolvable problem and allowed to choose either chip reward or noise punishment as feedback. It was found that subjects increase their probability of choosing noise over chips when the noise has been associated with control (S problems) and chips have been associated with a lack of control ( $\overline{\mathbf{S}}$ problems). The results were interpreted in terms of the properties of the noise as a signal for the existence of an S problem.
\end{abstract}

Several studies have indicated that subjects, when given a choice between situations in which they can exhibit control over events and those in which control is not possible, will choose control (see Miller, 1979, 1980, for reviews). In addition to conditions of actual control, preference for control extends to circumstances in which control is potential, that is, to situations in which subjects believe that they have the ability to modify an event, even though the control is never exercised (see Miller, 1980, for a review). Not surprisingly, subjects also express a preference for reward over punishment when both are available (Herman \& Azrin, 1964), even if the amount of reward for the reinforced response is substantially reduced from previous levels (Fantino, 1973).

The purpose of the present experiment was to investigate the preference for reward over punishment under conditions in which control was manipulated. In the present study, control was operationally defined as a subject's having the potential to solve conceptual problems. That is, subjects who are given solvable (S) problems are presumed to experience control, whereas subjects given unsolvable $(\overline{\mathrm{S}})$ problems are presumed to experience a lack of control. It was predicted that the probability that reward would be preferred to punishment would be a function of the history of control associated with reward and punishment, respectively, and, specifically, that the probability of choosing punishment over reward would increase as punishment became asso-

Requests for reprints should be sent to William J. Thomson, Department of Behavioral Sciences, University of Michigan, 4901 Evergreen Road, Dearborn, Michigan 48128. ciated with control and reward became associated with a lack of control.

\section{METHOD}

\section{Subjects}

The subjects were 64 introductory psychology students participating in partial fulfillment of a course requirement. Each subject was paid a participation fee of $\$ 2.00$, in addition to money earned in the experiment. Eight females and eight males were assigned randomly to each of the four experimental groups.

\section{Apparatus}

Each subject was presented with an identical series of three concept identification problems to solve. For the first two tasks, a set of stimuli composed of geometric forms of eight binary dimensions was constructed. The dimensions were: color of border (black, white), position of figure (left, right), number of figures (one, two), shade of background (light gray, dark gray), size of figure (large, small), shape of figure (ellipse, rectangle), color of figure (red, blue), and rotation of figure (vertical, horizontal). For Problem 1, the first four dimensions were varied and the latter four were held constant; in Problem 2, the first four dimensions were held constant and the latter four were varied. Stimuli were presented as slides in the same random order for each subject, with the restriction that a single stimulus could not appear in both Problem 1 and Problem 2 (i.e., all subjects saw exactly the same slides in exactly the same order). Extensive pilot data were used to construct two problems that were comparable in solvability and structure, with as little overlap in stimulus similarity as possible. Problem 3 consisted of stimuli composed of geometric forms with three dimensions: shape (circle, cross, square, hexagon), color (red, blue, green, yellow), and position of a black dot relative to the figure (above, below, left, right).

Performance feedback was given for each trial in each of the three problems by means of a green (correct) or red (error) light mounted on the subject's response panel. Furthermore, on certain trials, the subject was given additional feedback in the form 
of either a poker chip worth $\$ .005$ for a correct response or a taped 5-sec 90-dB burst of white noise presented through calibrated Maico earphones following an error. In addition to the feedback lights, the subject's response panel contained a white light that signaled the start of a trial and four push buttons through which the subject indicated a response, and,' in Problem 3 , a choice of the type of additional feedback desired.

\section{Procedure}

Four groups differing in problem solvability and feedback pattern on the first two concept identification tasks were constructed. A problem was defined as solvable (S) if there existed a single relevant dimension with consistent feedback. However, to ensure that all subjects had at least a minimal exposure to the noise feedback, each solvable problem began with three trials of error feedback, independently of the subjects' responses. Following these initial three trials, the relevant dimension for Problem 1 was color of border and for Problem 2, size. An unsolvable $(\overline{\mathrm{S}})$ problem was one in which the "correct" response was determined randomly for each trial. The order of "correct" responses on unsolvable problems was identical for all subjects.

On any trial, in addition to light feedback, the subject also received either a poker-chip reward $(\mathrm{C})$ if correct and no additional feedback if incorrect, or punishment in the form of noise (N) if in error and no additional feedback if correct. Thus, for each of the initial two problems, four conditions were possible: SC, SN, $\overline{\mathrm{S} C}$, and $\overline{\mathrm{S}} \mathrm{N}$. In Group 1, half of the subjects received Condition SC on Problem 1 and Condition $\bar{S} N$ on Problem 2, and the remaining subjects received $\overline{\mathrm{S} N}$ on Problem 1 and SC on Problem 2. With an analogous distribution of subjects, Group 2 received $\mathrm{SN}$ on one problem and $\overline{\mathrm{S} C}$ on the other, Group 3 received $\mathrm{SC}$ and $\mathrm{SN}$, and Group 4 received $\overline{\mathrm{S} C}$ and $\overline{\mathrm{SN}}$. Problem 3 was unsolvable and identical for all groups.

The subjects were read detailed instructions indicating that the experiment involved an investigation of the effects of reward and punishment on the learning of classifications. They were told that they would be given three problems to solve, and that some would involve receiving money for correct answers and others would involve hearing a loud noise when a response was incorrect. The stimuli for Problem 1 were then described, and the subject was told to try to learn a simple rule that would enable the correct classification of each slide. The task was described as "moderately difficult," and a description of the applicable C or $\mathrm{N}$ feedback was given. Immediately upon completion of Problem 1, Problem 2 was described and concluded in an analogous manner. Under SC and SN conditions, the subjects were run to a criterion of 10 consecutive correct responses; under $\overline{\mathrm{S} C}$ and $\overline{\mathrm{S} N}$ conditions, the subjects were run for a total of 64 trials. A total of five subjects (two in each of Groups 1 and 2, and one in Group 3) failed to reach criterion on solvable problems within 64 trials and were replaced.

For the third task, the subject was instructed that the problem was "similar to, but more difficult than" the preceding two tasks. Again, dimensions were described and a sample slide was shown. The subject was then told that a choice between $\mathrm{C}$ or $\mathrm{N}$ additional feedback would be allowed for each pair of trials, and that the subject was to indicate the desired option by pressing the appropriate button on the response panel. Instructions relating to which option to choose were left deliberately vague to allow the subject the greatest possible flexibility in choosing between the $\mathrm{C}$ and $\mathrm{N}$ alternatives. With the exception of the subject's choice of additional feedback and the corresponding action by the experimenter, the third problem proceeded in a manner identical to that of the first two. Since Problem 3 was unsolvable, each subject was run for a total of 36 pairs of trials.

\section{RESULTS}

The subjects were required to make a total of 36 choices between $\mathrm{C}$ or $\mathrm{N}$ additional feedback on Problem 3 , and the mean numbers of $\mathrm{N}$ choices were found to be 2.6,18.1, 6.9, and 5.2 for Groups $1,2,3$, and 4, respectively. An analysis of variance indicated a significant effect $[F(3,60)=13.26, p<.001]$, and all pairwise differences between means were examined further using the Sheffé multiple comparison procedure. These tests indicated that the mean of Group 2 differed significantly from each of the other group means [Sheffé critical value $(\mathrm{SCV})=9.38, \mathrm{p}<.01]$, with none of the other pairwise comparisons reaching significance.

In order to check for the possibility of differential learning as a function of $\mathrm{C}$ or $\mathrm{N}$ feedback, an analysis was performed to compare the total errors (TE) on Problem 1 for the subjects receiving $C$ feedback (half of Groups 1 and 3) with the TE for the subjects initially receiving $\mathrm{N}$ feedback (half of Group 2 plus the remaining subjects in Group 3). The difference between the mean values of 6.5 for the $C$ subcondition and 7.9 for the $\mathrm{N}$ subcondition failed to reach significance $[\mathrm{t}(30)=$ $.78, \mathrm{p}>.10]$.

Two analyses considered the effects of prior exposure to an $\overline{\mathrm{S}}$ problem on subsequent performance on an $\mathrm{S}$ problem. The first comparison considered the TE on Problem 2 for subjects receiving $\mathrm{C}$ feedback on that problem, conditional upon whether the initial problem was S (half of Group 3) or $\overline{\mathrm{S}}$ (half of Group 1). For each of these subjects, Problem 1 necessarily incorporated $\mathrm{N}$ feedback. No significant difference was found between the mean values of 8.4 ( $\overline{\mathrm{S}}$ subcondition) and 10.4 (S subcondition) $[\mathrm{t}(14)=.61, \mathrm{p}>.10]$. The second analysis was a similar comparison for TE on Problem 2 for subjects receiving $\mathrm{N}$ feedback on Problem 2, following an S Problem 1 (half of Group 3) or S Problem 1 (half of Group 2). For all of these subjects, Problem 1 utilized $\mathrm{C}$ feedback. Again, the mean values of 11.3 ( $\overline{\mathrm{S}}$ subcondition) and 7.9 (S subcondition) failed to reach significance $[\mathrm{t}(14)=1.53, \mathrm{p}>.10]$.

\section{DISCUSSION}

The major finding of this study is that, within the parameters of this experiment, subjects are relatively more likely to choose punishment over reward when punishment has been associated with control and when reward has been associated with a lack of control. It should be noted that the data do not support the interpretation that subjects prefer punishment on an absolute basis, since punishment is chosen, at most, only approximately $50 \%$ of the time (Group 2). When control is held constant or is associated with reward, subjects overwhelmingly prefer reward.

Explanation of the results involves a consideration of the chips and noise as discriminative cues for problem solution, and thus for intrinsic reward (see Deci, 1975, and Deci \& Porac, 1978, for a discussion of intrinsic and extrinsic reward). This approach emphasizes the signaling properties of an event for a subsequent reward and proposes that subjects will choose chips or noise based on their properties as differential signals for intrinsic reward. Thus, in Groups 3 and 4, since neither the chips nor the noise are differential predictors of problem solvability, subjects might be predicted to choose chips based on a general preference for reward. However, for both remaining groups there exists a reliable signal for problem solvability, and therefore subjects would be predicted to prefer chips (Group 1) or noise (Group 2) because of their properties as discriminative 
cues. Preference for a noxious stimulus because of its signaling properties has been demonstrated by Holz and Azrin (1961), who trained pigeons to respond in the presence of shock by pairing the shock with a food reward, followed by trials in which both shock and food were absent. This procedure led to increased responding when the shock was present and a corresponding decrease when the shock was absent. Similar results emphasizing the role of noxious stimuli as discriminative cues have been reported by Fowler (1971) and Fowler and Wischner (1969). Thus, in the present experiment, Group 2 subjects may have learned that noise was a signal that the problem was solvable and, conversely, that the chips signaled an unsolvable problem.

In conclusion, the present study demonstrates an increased likelihood that subjects will choose punishment over reward when punishment has been associated with control and reward with a lack of control. However, it should be noted that both the rewards and punishments utilized in this study were mild. As stimulus intensity is increased, primary properties of the stimulus as a reward or punishment may supercede the characteristics of the stimulus as a discriminative signal for intrinsic reward.

\section{REFERENCES}

Deci, E. L. Intrinsic motivation. New York: Plenum, 1975.

Deci, E. L., \& Porac, J. Cognitive evaluation theory and the study of human motivation. In M. R. Lepper \& D. Greene (Eds.), The hidden costs of reward. Hillsdale, N.J: Erlbaum, 1978.

Fantino, E. Aversive control. In J. A. Nevin \& G. S. Reynolds (Eds.), The study of behavior. Glenview, Ill: Scott, Foresman, 1973.

Fowler, H. Suppression and facilitation by response contingent shock. In F. R. Brush (Ed.), A versive conditioning and learning. New York: Academic Press, 1971.

Fowler, H., \& Wischner, G. J. The varied function of punishment in discrimination learning. In B. A. Campbell \& R. M. Church (Eds.), Punishment and aversive behavior. New York: Appleton-Century-Crofts, 1969.

Herman, R. L., \& Azrin, N. H. Punishment by noise in an alternative response situation. Journal of the Experimental Analysis of Behavior, 1964, 7, 185-188.

Holz, W. C., \& Azrin, N. H. Discrimination properties of punishment. Journal of the Experimental Analysis of Behavior, 1961, 4, 225-232.

Miller, S. M. Controllability and human stress: Method, evidence and theory. Behavior Research and Therapy, 1979, 17, 287-304.

Miller, S. M. Why having control reduces stress: If I can stop the roller coaster, I don't want to get off. In J. Garber \& M. E. P. Seligman (Eds.), Human helplessness: Theory and applications. New York: Academic Press, 1980.

(Manuscript received for publication August 15, 1983.) 